\title{
Evaluation of Cow Based Fermented Organic Products for Non-insecticidal Pest Management in Castor
}

\author{
G. V. Suneel Kumar* and O. Sarada \\ Acharya N. G. Ranga Agril. University, Lam, Guntur, Andhra Pradesh, India \\ *Corresponding author
}

\section{A B S T R A C T}

\section{Keywords}

Castor, cow based organics, NSKE,

Sucking and Defoliator insect pests

\section{Article Info}

Accepted:

04 September 2020 Available Online: 10 October 2020
Field studies were undertaken at Agricultural Research Farm, Darsi, Andhra Pradesh to study the comparative bio-efficacy of cow based (dung and urine) fermented organic products viz., neemastra, brahmastra and agniastra at $20 \%$ along with Neem Seed Kernel Extract (NSKE) $5 \%$ against the major pests of castor and their safety to natural enemies during 2016-17. NSKE 5\% recorded least no. of mean larval population of Achaea janata (1.06 plant $\left.^{-1}\right)$, Spodoptera litura $\left(0.78\right.$ plant $\left.^{-1}\right)$, capsule damage by Conogethes punctiferalis $(17.0 \%)$ and was no way inferior to recommended insecticidal check treatment $\left(0.74,0.66\right.$ larvae plant ${ }^{-1}$ and $11.7 \%$, respectively). NSKE @ 5\% and neemastra @ 20\% registered significantly least (2.15 and 2.40 hoppers leaf $^{-1}$ ) population of Empoasca flavescens and were on par with the other organics including insecticidal check (2.63 leaf ${ }^{1}$ ) but superior to untreated control $\left(3.8\right.$ leaf $\left.^{-1}\right)$. NSKE $5 \%$ followed by neemastra, brahmastra and agniastra conserved highest population of coccinellids $(1.3,1.1,1.0$ and 0.96 plant $^{-1}$, respectively), spiders $\left(1.0,1.0,1.0\right.$ and 1.08 plant $^{-1}$, respectively) and Microplitis coccons $\left(1.34,1.15,1.11\right.$ and 1.1 plant $^{-1}$, respectively) which were at par with untreated control $\left(1.5,1.3\right.$ and 1.5 plant $^{-1}$, respectively). NSKE 5\% recorded higher seed yield of $742 \mathrm{~kg} \mathrm{ha}^{-1}$ with ICBR of 16.4 followed by agniastra (474 $\left.\mathrm{kg} \mathrm{ha}^{-1}\right)$ with ICBR of 3.4 and were next best to insecticidal check $\left(885 \mathrm{~kg} \mathrm{ha}^{-1}\right)$ recorded ICBR of 10.2.

\section{Introduction}

Castor, Ricinus communis Linnaeus, is an important industrially valued non edible oilseed crop and is grown especially in arid and semi arid regions. India is the largest producer of castor seed and oil, which account for 5 per cent area and 64 per cent production in the world (Mukesh et al., 2016). Castor crop suffers from many biotic stresses and among them defoliator pests'viz. semilooper, Achaea janata L., tobacco caterpillar, Spodoptera litura (F.), shoot and capsule borer, Conogethes punctiferalis Guen. and sucking insect, leafhopper, Empoasca flavescens (F.) are of economic importance (Shilpakala and Murali Krishna, 2016). The control of insect pests in rainfed castor mainly involves pesticides, but it has many drawbacks. This situation warranted developing cost effective, environmentally friendly and safe pest control strategies to mitigate the problems created by chemical control. Historically, organic wastes of animal 
origin were most commonly used for nutrition and plant protection. In addition, herbs processed in liquid excreta of animals were used for plant protection (Kavitha, 2009). In this direction, scientific evaluation of biodynamic pesticides, botanicals and biorationals including indigenous technologies are considered very much essential to combat noxious pests of rainfed castor in the dryland tracts of Andhra Pradesh.

The information on environmentally safe and biodegradable non-pesticidal pest management options in castor under rainfed condition is scanty. Hence, present investigation was intended to identify the best cow based organic alternatives for noninsecticidal pest management in castor with the following objectives include to study the bio-efficacy of cow based fermented organics for non-insecticidal pest management in castor. To ascertain the effect of different cow based fermented organics on natural enemies of castor pests in the field condition. And also to know the cost effectiveness of cow based fermented organics in castor pest management

\section{Materials and Methods}

A field experiment was conducted in the research farm of Agricultural Research Station, Darsi under the aegis of Acharya N. G. Ranga Agricultural University, Andhra Pradesh during 2016-17 to evaluate the bioefficacy of three cow based fermented organics viz., neemastra, bramhastra and agniastra. For the purpose, popular castor hybrid, PCH 111 was sown with a spacing of $90 \times 60 \mathrm{~cm}$. in randomized block design with four replications having uniform plot size of $7.0 \mathrm{~m} \mathrm{x} 6.0 \mathrm{~m}$. All the recommended agronomical practices except plant protection were followed as and when required. Considering the pest population in experimental area, two sprays were imposed on need basis. Treatment details include the following.

\begin{tabular}{|c|c|c|}
\hline Treatments & Composition & Dosage \\
\hline Neemastra & $\begin{array}{l}100 \text { lt of water }+5 \mathrm{lt} \text { of gomuthra }+5 \mathrm{~kg} \text { of } \\
\text { cow dung }+5 \mathrm{~kg} \text { of neem leaf pulp }\end{array}$ & $\begin{array}{l}2 \text { lt of extract in } 100 \mathrm{lt} \\
\text { of water }(20 \%)\end{array}$ \\
\hline Bramhastra & $\begin{array}{l}2 \mathrm{~kg} \text { each of neem, sitaphal, Calotropis, } \\
\text { pongamia, guava leaf pulp in } 10 \mathrm{lt} \text { of } \\
\text { gomuthra }\end{array}$ & $\begin{array}{l}2 \text { lt of extract in } 100 \mathrm{lt} \\
\text { of water }(20 \%)\end{array}$ \\
\hline Agniastra & $\begin{array}{l}1 \mathrm{~kg} \text { tobacco }+0.5 \mathrm{~kg} \text { green chilli pulp }+0.5 \\
\mathrm{~kg} \text { garlic pulp }+5 \mathrm{~kg} \text { neem leaf pulp }+10 \mathrm{lt} \text { of } \\
\text { gomuthra }\end{array}$ & $\begin{array}{l}2 \text { lt of extract in } 100 \mathrm{lt} \\
\text { of water }(20 \%)\end{array}$ \\
\hline $\begin{array}{l}\text { Neem Seed Kernel Extract } \\
\text { (organic check) }\end{array}$ & $\begin{array}{l}5 \mathrm{~kg} \text { Neem seed kernel powder }+10 \text { lt of } \\
\text { water }\end{array}$ & $\begin{array}{l}1 \text { lt of extract in } 10 \text { lt of } \\
\text { water }(5 \%)\end{array}$ \\
\hline $\begin{array}{l}\text { Acephate followed by Thiodicarb } \\
\text { (recommended insecticidal } \\
\text { check) }\end{array}$ & Readily available insecticide formulations & $\begin{array}{l}1 \mathrm{gm} \text { each in } 1 \mathrm{lt} \text { of } \\
\text { water }(0.1 \%)\end{array}$ \\
\hline Untreated Check (Water spray) & -- & -- \\
\hline
\end{tabular}

The observations on pest and natural enemies' viz., coccinellid grubs and adults, spiders and Micropletis cocoons on $A$. janata larvae were made on five random plants in each plot at 5, 10 and 15 days after each spray. Infestation of semilooper, tobacco caterpillar was assessed by recording the number of larvae per plant.
Infestation of shoot and capsule borer was assessed by recording the number of total and affected capsules on each of the five randomly selected plants per treatment and per cent infestation of capsules for each plant was calculated. Number of nymphs and adult population of leafhopper on top three leaves 
per plant were recorded. Overall mean harmful, beneficial insect numbers and percent capsule damage across sampling intervals was determined for making treatment wise comparisons.

Plot wise castor seed yields were computed on hectare basis and were subjected to suitable transformations for statistical analysis (Gomez and Gomez, 1984). Incremental Cost Benefit Ratio (ICBR) was worked out to know the economics of each treatment.

\section{Results and Discussion}

\section{Evaluation of cow based organics against castor semilooper}

Mean of three sampling intervals $(5,10$ and 15 days) after the first spraying (Table 1) revealed that significant difference in larval population of $A$. janata between the treatments and lowest larval incidence was observed in recommended insecticide check, acephate (0.94 larvae plant $\left.{ }^{-1}\right)$ followed by NSKE@5\% (1.22 larvae plant $\left.{ }^{-1}\right)$ which is at par with agniastra @20\% (1.48 larvae plant $\left.{ }^{-1}\right)$ and neemastra@20\% (2.02 larvae plant $\left.{ }^{-1}\right)$.

Brahmastra@20\% was statistically at par with former three treatments by recording 2.12 larvae plant $^{-1}$ and significantly different from untreated control (3.80 larvae plant ${ }^{-1}$ ). Mean of observations (5, 10 and 15 days) recorded after second spraying revealed that lowest population was recorded in thiodicarb (0.54 larvae plant $\left.{ }^{-1}\right)$ followed by NSKE @ 5\% (0.90 larvae plant $^{-1}$ ) and were significantly different from remaining treatments.

Agniastra@20\% was next best treatment to record 3.12 larvae plant $^{-1}$ which was on par with brahmastra@20\% (3.36 larvae plant ${ }^{-1}$ ) and neemastra@20\% (3.70 larvae plant $\left.{ }^{-1}\right)$. The latter two treatments were statistically at par with untreated check (4.48 larvae plant ${ }^{-1}$ ).
Cumulative mean of two sprays (Table 1) showed that NSKE@5\% recorded at par larval population of A. janata (1.06 larvae plant $^{-1}$ ) with recommended insecticide check treatment, acephate at first spray followed by second spray of thiodicarb (0.74 larvae plant $\left.^{-1}\right)$.

All the cow based organics viz., agniastra, brahmastra and neemastra @ 20\% recorded at par populations of $A$. janata (2.30, 2.74 and 2.86 larvae plant $^{-1}$, respectively) compared to untreated control which recorded significantly higher larval population of 4.14 semilooper plant $^{-1}$.

\section{Evaluation of cow based organics against tobacco caterpillar}

After first spraying, insecticide check, acephate showed its superiority in recording mean (5, 10 and 15 days sampling points) lowest larval population (0.90 plant $\left.^{-1}\right)$ of $S$. litura and was at par with NSKE 5\% (1.02 larvae plant $\left.{ }^{-1}\right)$. Brahmastra, agniastra and neemastra@20\% treatments were next in the order of efficacy with at par larval populations of $1.75,1.95$ and 2.09 larvae plant $^{-1}$, respectively. The untreated check recorded significantly highest population of 4.22 larvae plant ${ }^{-1}$ (Table 1).

After second spraying, the chemical check, thiodicarb (0.42 larvae plant $\left.{ }^{-1}\right)$ and NSKE 5\% (0.54 larvae plant $\left.^{-1}\right)$ maintained their superiority in recording mean $(5,10$ and 15 days observations) lowest larval population of $S$. litura and differed significantly with the remaining treatments.

Agniastra@20\% (1.85 larvae plant ${ }^{-1}$ ) was significantly different from brahmastra @ 20\% and neemastra@ 20\% (3.13 and 3.31 larvae plant ${ }^{-1}$, respectively) which were at par with each other but significantly different from untreated control (4.62 larvae plant ${ }^{-1}$ ). 
Table.1 Efficacy of cow based organics against major insect pests infesting castor during 2016-17

\begin{tabular}{|c|c|c|c|c|c|c|c|c|c|c|c|c|}
\hline \multirow[t]{2}{*}{ Treatments } & \multicolumn{3}{|c|}{$\begin{array}{c}\text { *Mean no. of } A . \text { janata larvae/ } \\
\text { plant }\end{array}$} & \multicolumn{3}{|c|}{$\begin{array}{c}\text { *Mean no. of } S . \text { litura larvae/ } \\
\text { plant }\end{array}$} & \multicolumn{3}{|c|}{$\begin{array}{c}* * \text { Mean Shoot and Capsule } \\
\text { borer damage }(\%)\end{array}$} & \multicolumn{3}{|c|}{$\begin{array}{l}\text { *Mean population of Leaf } \\
\text { hopper/ leaf }\end{array}$} \\
\hline & $\begin{array}{l}\text { After } \mathbf{I}^{\text {st }} \\
\text { spray }\end{array}$ & $\begin{array}{l}\text { After } \\
\text { II }^{\text {nd }} \\
\text { spray }\end{array}$ & $\begin{array}{l}\text { Mean of } \\
\text { two } \\
\text { sprays }\end{array}$ & $\begin{array}{l}\text { After } \mathbf{I}^{\text {st }} \\
\text { spray }\end{array}$ & $\begin{array}{l}\text { After } \\
\text { II }^{\text {nd }} \\
\text { spray }\end{array}$ & $\begin{array}{l}\text { Mean of } \\
\text { two } \\
\text { sprays }\end{array}$ & $\begin{array}{l}\text { After I } I^{\text {st }} \\
\text { spray }\end{array}$ & $\begin{array}{l}\text { After } \\
\text { II }^{\text {nd }} \\
\text { spray }\end{array}$ & $\begin{array}{l}\text { Mean of } \\
\text { two } \\
\text { sprays }\end{array}$ & $\begin{array}{l}\text { After } \mathbf{I}^{\text {st }} \\
\text { spray }\end{array}$ & $\begin{array}{l}{\text { After } \text { II }^{\text {nd }}}_{\text {spray }}\end{array}$ & $\begin{array}{c}\text { Mean of } \\
\text { two } \\
\text { sprays }\end{array}$ \\
\hline Neemastra at $20 \%$ & $\begin{array}{c}2.02 \\
(1.40)^{\mathrm{bc}}\end{array}$ & $\begin{array}{c}3.70 \\
(1.91)^{\mathrm{ab}}\end{array}$ & $\begin{array}{c}2.86 \\
(1.68)^{\mathrm{b}}\end{array}$ & $\begin{array}{c}2.09 \\
(1.42)^{\mathrm{b}}\end{array}$ & $\begin{array}{c}3.31 \\
(1.81)^{\mathrm{b}}\end{array}$ & $\begin{array}{c}2.70 \\
(1.64)^{b}\end{array}$ & $\begin{array}{c}20.18 \\
(26.68)^{\mathrm{bc}}\end{array}$ & $\begin{array}{c}28.23 \\
(32.07)^{\mathrm{c}}\end{array}$ & $\begin{array}{c}24.20 \\
(29.45)^{\mathrm{c}}\end{array}$ & $\begin{array}{c}2.55 \\
(1.60)^{\mathrm{bcd}}\end{array}$ & $\begin{array}{c}2.25 \\
(1.49)^{\mathrm{cd}}\end{array}$ & $\begin{array}{c}2.40 \\
(1.54)^{\mathrm{c}}\end{array}$ \\
\hline Agniastra at $20 \%$ & $\begin{array}{c}1.48 \\
(1.20)^{\mathrm{bc}}\end{array}$ & $\begin{array}{c}3.12 \\
(1.75)^{b}\end{array}$ & $\begin{array}{c}2.30 \\
(1.51)^{\mathrm{b}}\end{array}$ & $\begin{array}{c}1.95 \\
(1.39)^{\mathrm{b}}\end{array}$ & $\begin{array}{c}1.85 \\
(1.36)^{\mathrm{c}}\end{array}$ & $\begin{array}{c}1.90 \\
(1.38)^{\mathrm{c}}\end{array}$ & $\begin{array}{c}18.40 \\
(25.40)^{\mathrm{c}}\end{array}$ & $\begin{array}{c}28.80 \\
(32.45)^{\mathrm{c}}\end{array}$ & $\begin{array}{c}23.60 \\
(29.05)^{\mathrm{c}}\end{array}$ & $\begin{array}{c}3.30 \\
(1.81)^{\mathrm{ab}}\end{array}$ & $\begin{array}{c}2.80 \\
(1.67)^{\mathrm{bc}}\end{array}$ & $\begin{array}{c}3.05 \\
(1.74)^{b}\end{array}$ \\
\hline NSKE 5\% & $\begin{array}{c}1.22 \\
(1.08)^{\mathrm{bc}}\end{array}$ & $\begin{array}{c}0.90 \\
(0.92)^{\mathrm{c}}\end{array}$ & $\begin{array}{c}1.06 \\
(1.02)^{\mathrm{c}}\end{array}$ & $\begin{array}{c}1.02 \\
(1.00)^{\mathrm{cd}}\end{array}$ & $\begin{array}{c}0.54 \\
(0.72)^{d}\end{array}$ & $\begin{array}{c}0.78 \\
(0.87)^{d}\end{array}$ & $\begin{array}{c}12.90 \\
(21.05)^{\mathrm{d}}\end{array}$ & $\begin{array}{c}21.10 \\
(27.31)^{\mathrm{d}}\end{array}$ & $\begin{array}{c}17.00 \\
(24.35)^{\mathrm{d}}\end{array}$ & $\begin{array}{c}2.25 \\
(1.49)^{\mathrm{cd}}\end{array}$ & $\begin{array}{c}2.05 \\
(1.42)^{d}\end{array}$ & $\begin{array}{c}2.15 \\
(1.46)^{c}\end{array}$ \\
\hline $\begin{array}{l}\text { Acephate } 0.1 \% \text { at } I^{\text {st }} \\
\text { spray and Thiodicarb } \\
0.1 \% \text { at } I^{\text {nd }} \text { spray }\end{array}$ & $\begin{array}{c}0.94 \\
(0.96)^{\mathrm{c}}\end{array}$ & $\begin{array}{c}0.54 \\
(0.69)^{\mathrm{c}}\end{array}$ & $\begin{array}{c}0.74 \\
(0.85)^{\mathrm{c}}\end{array}$ & $\begin{array}{c}0.90 \\
(0.93)^{\mathrm{d}}\end{array}$ & $\begin{array}{c}0.42 \\
(0.64)^{\mathrm{d}}\end{array}$ & $\begin{array}{c}0.66 \\
(0.81)^{\mathrm{d}}\end{array}$ & $\begin{array}{c}9.80 \\
(18.23)^{\mathrm{e}}\end{array}$ & $\begin{array}{c}13.60 \\
(21.59)^{\mathrm{e}}\end{array}$ & $\begin{array}{c}11.70 \\
(19.98)^{\mathrm{e}}\end{array}$ & $\begin{array}{c}2.10 \\
(1.45)^{\mathrm{d}}\end{array}$ & $\begin{array}{c}3.15 \\
(1.76)^{b}\end{array}$ & $\begin{array}{c}2.63 \\
(1.62)^{\mathrm{bc}}\end{array}$ \\
\hline Untreated check & $\begin{array}{c}3.80 \\
(1.93)^{\mathrm{a}}\end{array}$ & $\begin{array}{c}4.48 \\
(2.11)^{\mathrm{a}}\end{array}$ & $\begin{array}{c}4.14 \\
(2.02)^{\mathrm{a}}\end{array}$ & $\begin{array}{c}4.22 \\
(2.04)^{\mathrm{a}}\end{array}$ & $\begin{array}{c}4.62 \\
(2.14)^{\mathrm{a}}\end{array}$ & $\begin{array}{c}4.42 \\
(2.10)^{\mathrm{a}}\end{array}$ & $\begin{array}{c}22.40 \\
(28.23)^{\mathrm{a}}\end{array}$ & $\begin{array}{c}47.60 \\
(43.62)^{\mathrm{a}}\end{array}$ & $\begin{array}{c}35.00 \\
(36.26)^{\mathrm{a}}\end{array}$ & $\begin{array}{c}3.70 \\
(1.93)^{\mathrm{a}}\end{array}$ & $\begin{array}{c}3.90 \\
(1.97)^{\mathrm{a}}\end{array}$ & $\begin{array}{c}3.80 \\
(1.95)^{\mathrm{a}}\end{array}$ \\
\hline CV $(\%)$ & 22.9 & 14.7 & 13.5 & 15.9 & 9.62 & 9.39 & 3.80 & 4.22 & 3.43 & 9.17 & 8.29 & 6.87 \\
\hline CD (0.05) & 0.46 & 0.34 & 0.30 & 0.32 & 0.21 & 0.20 & 1.39 & 2.03 & 1.47 & 0.23 & 0.20 & 0.17 \\
\hline
\end{tabular}

Mean of four replications

*Values in parenthesis are SQRT values, **Values in parenthesis are arc sine values

NSKE - Neem Seed Kernel Extract

In a column, means followed by a common letter (s) are not significantly different by DMRT $(\mathrm{P}=0.05)$ 
Table.2 Effect of cow based organics on natural enemies of insect pests and seed yields in castor during 2016-17

\begin{tabular}{|c|c|c|c|c|c|c|c|c|c|c|c|c|c|}
\hline \multirow[t]{2}{*}{ Treatments } & \multicolumn{3}{|c|}{ *Mean no. of Coccinellids/ plant } & \multicolumn{3}{|c|}{ *Mean no. of Spiders/ plant } & \multicolumn{3}{|c|}{$\begin{array}{l}\text { * Mean no. of } A . \text { janata larvae } \\
\text { with Microplitis cocoons }\end{array}$} & \multirow{2}{*}{$\begin{array}{c}\text { Seed } \\
\text { Yield } \\
\text { (Kg/ha } \\
\text { ) }\end{array}$} & \multirow{2}{*}{$\begin{array}{l}\text { Additional } \\
\text { returns } \\
\text { (₹ /ha) }\end{array}$} & \multirow{2}{*}{$\begin{array}{c}\text { Net } \\
\text { returns } \\
(₹ / h a)\end{array}$} & \multirow[t]{2}{*}{ ICBR } \\
\hline & $\begin{array}{c}\text { After } \mathbf{I}^{\text {st }} \\
\text { spray }\end{array}$ & $\begin{array}{l}\text { After } \\
\text { II }^{\text {nd }} \\
\text { spray }\end{array}$ & $\begin{array}{c}\text { Mean of } \\
\text { two } \\
\text { sprays }\end{array}$ & $\begin{array}{l}\text { After I } \\
\text { spray }\end{array}$ & $\begin{array}{l}\text { After } \\
\text { II }^{\text {nd }} \\
\text { spray }\end{array}$ & $\begin{array}{c}\text { Mean of } \\
\text { two } \\
\text { sprays }\end{array}$ & $\begin{array}{l}\text { After } \mathbf{I}^{\text {st }} \\
\text { spray }\end{array}$ & $\begin{array}{l}\text { After } \\
\text { II }^{\text {nd }} \\
\text { spray }\end{array}$ & $\begin{array}{c}\text { Mean of } \\
\text { two } \\
\text { sprays }\end{array}$ & & & & \\
\hline $\begin{array}{l}\text { Neemastra at } \\
20 \%\end{array}$ & $\begin{array}{c}1.20 \\
(1.31)^{\mathrm{ab}}\end{array}$ & $\begin{array}{c}1.00 \\
(0.99)^{\mathrm{a}}\end{array}$ & $\begin{array}{c}1.10 \\
(1.05)^{\mathrm{ab}}\end{array}$ & $\begin{array}{c}0.90 \\
(0.94)^{\mathrm{a}}\end{array}$ & $\begin{array}{c}1.10 \\
(1.04)^{\mathrm{a}}\end{array}$ & $\begin{array}{c}1.00 \\
(0.99)^{\mathrm{a}}\end{array}$ & $\begin{array}{c}0.95 \\
(1.19)^{\mathrm{a}}\end{array}$ & $\begin{array}{c}1.35 \\
(1.35)^{\mathrm{a}}\end{array}$ & $\begin{array}{c}1.15 \\
(1.29)^{\mathrm{b}}\end{array}$ & $392^{\text {de }}$ & 1360 & 510 & 0.60 \\
\hline $\begin{array}{l}\text { Bramhastra at } \\
20 \%\end{array}$ & $\begin{array}{c}1.00 \\
(1.22)^{\mathrm{b}}\end{array}$ & $\begin{array}{c}1.00 \\
(0.99)^{\mathrm{a}}\end{array}$ & $\begin{array}{c}1.00 \\
(1.00)^{\mathrm{b}}\end{array}$ & $\begin{array}{c}0.80 \\
(0.89)^{\mathrm{a}}\end{array}$ & $\begin{array}{c}1.20 \\
(1.09)^{\mathrm{a}}\end{array}$ & $\begin{array}{c}1.00 \\
(0.99)^{\mathrm{a}}\end{array}$ & $\begin{array}{c}1.03 \\
(1.22)^{\mathrm{a}}\end{array}$ & $\begin{array}{c}1.20 \\
(1.28)^{\mathrm{a}}\end{array}$ & $\begin{array}{c}1.11 \\
(1.27)^{\mathrm{b}}\end{array}$ & $419^{d}$ & 2480 & 1630 & 1.92 \\
\hline $\begin{array}{l}\text { Agniastra at } \\
20 \%\end{array}$ & $\begin{array}{c}0.93 \\
(1.19)^{\mathrm{b}}\end{array}$ & $\begin{array}{c}1.00 \\
(1.00)^{\mathrm{a}}\end{array}$ & $\begin{array}{c}0.96 \\
(0.99)^{\mathrm{b}}\end{array}$ & $\begin{array}{c}1.03 \\
(1.01)^{\mathrm{a}}\end{array}$ & $\begin{array}{c}1.13 \\
(1.05)^{\mathrm{a}}\end{array}$ & $\begin{array}{c}1.08 \\
(1.04)^{\mathrm{a}}\end{array}$ & $\begin{array}{c}0.95 \\
(1.18)^{\mathrm{a}}\end{array}$ & $\begin{array}{c}1.25 \\
(1.32)^{\mathrm{a}}\end{array}$ & $\begin{array}{c}1.10 \\
(1.26)^{b}\end{array}$ & $474^{c}$ & 4640 & 3640 & 3.64 \\
\hline NSKE $5 \%$ & $\begin{array}{c}1.20 \\
(1.31)^{\mathrm{ab}}\end{array}$ & $\begin{array}{c}1.40 \\
(1.17)^{\mathrm{a}}\end{array}$ & $\begin{array}{c}1.30 \\
(1.14)^{\mathrm{ab}}\end{array}$ & $\begin{array}{c}0.80 \\
(0.90)^{\mathrm{a}}\end{array}$ & $\begin{array}{c}1.20 \\
(1.08)^{\mathrm{a}}\end{array}$ & $\begin{array}{c}1.00 \\
(0.99)^{\mathrm{a}}\end{array}$ & $\begin{array}{c}1.40 \\
(1.37)^{\mathrm{a}}\end{array}$ & $\begin{array}{c}1.60 \\
(1.45)^{\mathrm{a}}\end{array}$ & $\begin{array}{c}1.50 \\
(1.42)^{\mathrm{a}}\end{array}$ & $742^{b}$ & 15360 & 14485 & 16.55 \\
\hline Untreated check & $\begin{array}{c}1.60 \\
(1.44)^{\mathrm{a}}\end{array}$ & $\begin{array}{c}1.40 \\
(1.18)^{\mathrm{a}}\end{array}$ & $\begin{array}{c}1.50 \\
(1.21)^{\mathrm{a}}\end{array}$ & $\begin{array}{c}1.20 \\
(1.09)^{\mathrm{a}}\end{array}$ & $\begin{array}{c}1.40 \\
(1.18)^{\mathrm{a}}\end{array}$ & $\begin{array}{c}1.30 \\
(1.14)^{\mathrm{a}}\end{array}$ & $\begin{array}{c}1.30 \\
(1.34)^{\mathrm{a}}\end{array}$ & $\begin{array}{c}1.38 \\
(1.37)^{\mathrm{a}}\end{array}$ & $\begin{array}{c}1.34 \\
(1.35)^{\mathrm{ab}}\end{array}$ & $358^{\mathrm{e}}$ & -- & -- & -- \\
\hline $\mathrm{CV}(\%)$ & 9.27 & 17.15 & 13.81 & 15.25 & 16.67 & 11.64 & 13.15 & 10.69 & 6.10 & 5.12 & -- & -- & -- \\
\hline CD (0.05) & 0.17 & 0.26 & 0.20 & 0.20 & 0.25 & 0.17 & 0.23 & 0.21 & 0.12 & 42.1 & -- & -- & -- \\
\hline
\end{tabular}

Mean of four replications

*Values in parenthesis are SQRT values

NSKE - Neem Seed Kernel Extract

Market price of castor seed- ₹ 40/kg

In a column, means followed by a common letter (s) are not significantly different by DMRT $(\mathrm{P}=0.05)$ 
The overall mean larval populations of $S$. litura after two sprayings (Table 1) was significantly reduced by acephate at first spray followed by second spray of thiodicarb (0.66 larvae plant $\left.{ }^{-1}\right)$ and NSKE 5\% (0.78 larvae plant ${ }^{-1}$ ) compared with cow based fermented organic sprays. Agniastra @ 20\% recorded comparatively lower $S$. litura population (1.90 larvae plant $\left.{ }^{-1}\right)$ and was at par with brahmastra @20\% (2.44 larvae plant ${ }^{-1}$ ) which in turn at par with two sprays of neemastra @ 20\% (2.70 larvae plant $\left.{ }^{-1}\right)$.

The untreated check recorded significantly highest mean population of 4.42 larvae plant ${ }^{-1}$ (Table 1). NSKE 5\% was effective in reducing the defoliator population in castor due to various secondary metabolites in neem including azadirachtin which act as defensive weapon against herbivores. Further, the effectiveness of NSKE under field condition to bring down the pest population was reviewed by several workers in the past which agrees with the present findings.

\section{Evaluation of cow based organics against shoot and capsule borer}

At the time of spike development, mean data of three samplings at 5,10 and 15days after first spray showed that plots treated with insecticide check acephate registered minimum $(9.80 \%)$ capsule borer damage due to $C$. punctiferalis significantly followed by NSKE $5 \%(12.9 \%)$ which differed from rest of the treatments (Table 1). The next best treatment was observed to be agniastra @20\% (18.4\%) which was at par with neemastra @ 20\% (20.2\%) in turn with bramhastra @20\% $(20.5 \%)$ and proved significantly superior over untreated control $(22.4 \%)$. Mean results of three samplings at 5, 10 and 15 days after second spray showed similar trend in treatment efficacy, wherein recommended plant protection treatment thiodicarb (13.6\%) and NSKE @ 5\% (21.1\%) proved to be the best in reducing the capsule borer damage in castor. Cumulative mean results of two sprays (Table 1) showed that significantly least percentage of capsule damage (11.7) was registered in plots sprayed with acephate at first spray followed by second spray of thiodicarb over rest of the treatments. NSKE 5\% was also found to be better treatment next to recommended plant protection treatment in minimizing the capsule damage $(17.0 \%)$ due to $C$. punctiferalis. Agniastra (23.6\%) and Neemastra (24.2\%) sprayed at $20 \%$ concentration proved equally effective against the pest. Brahmastra @20\% recorded highest percentage of capsule damage (26.8) but proved significantly superior to untreated control $(35.0 \%)$.

\section{Evaluation of cow based organics against leaf hopper}

Mean of three sampling points $(5,10$ and 15 days) calculated after first spray (Table 1) indicated that least numbers of leaf hopper, $E$. flavescens (2.10 leaf $\left.{ }^{-1}\right)$ were registered in plots treated with acephate followed by NSKE 5\% (2.25 leaf $\left.{ }^{-1}\right)$. These two treatments exhibited significantly lesser incidence of $E$. flavescens and were at par with neemastra (2.55 hoppers leaf $^{-1}$ ) treatment. Other cow based fermented organics, brahmastra (3.00 hoppers leaf $^{-1}$ ) as well as agniastra (3.30 hoppers leaf ${ }^{-1}$ ) with $20 \%$ concentration also proved significantly superior against $E$. flavescens infesting castor over untreated control (3.70 hoppers leaf $\left.{ }^{1}\right)$. After second spraying, mean population of E. flavescens recorded at three samplings $(5,10$ and 15 days) showed that NSKE 5\% registered significantly minimum (2.05 leaf $\left.{ }^{-1}\right)$ population and was at par with brahmastra (2.20 leaf l $\left.^{-1}\right)$ and neemastra @ 20\% (2.25 leaf $\left.{ }^{-1}\right)$. Agniastra@20\% (2.80 hoppers leaf ${ }^{-1}$ ) recorded at par population with the former two fermented organics which was also on par with the insecticide check (3.15 hoppers 
leaf ${ }^{-1}$ ) and both were superior to untreated control (3.90 hoppers leaf $\left.{ }^{-1}\right)$.

Cumulative mean data on leaf hopper, $E$. flavescens population worked out for two sprays revealed that NSKE @5\% and neemastra @20\% registered significantly least (2.15 and 2.40 hoppers leaf ${ }^{-1}$ ) population of the pest (Table 1). Brahmastra @20\% (2.60 hoppers leaf ${ }^{-1}$ ) and recommended insecticide check i.e., acephate at first spray followed by second spray of thiodicarb (2.63 hoppers leaf $\left.{ }^{1}\right)$ proved at par efficacy with the former two treatments and also with agniastra (3.05 hoppers $\quad$ leaf $^{-1}$ ). Significantly higher population of E. flavescens was recorded in untreated control (3.80 hoppers leaf $\left.{ }^{-1}\right)$.

\section{Evaluation of cow based organics against beneficial insects}

Pooled data (Table 2) on natural enemies i.e. coccinellids (grubs + adults), spider population and $A$. janata larvae with Microplitis cocoons after three sampling points $(5,10$ and 15 days) of first and second sprays was homogenous in all the treatments except insecticidal application. Mean data of two sprays showed that NSKE 5\% (1.30, $1.00,1.50$ plant $\left.^{-1}\right)$ followed by neemastra $\left(1.10,1.00,1.15\right.$ plant $\left.^{-1}\right)$, brahmastra $(1.00$, 1.00 and 1.11 plant $\left.^{-1}\right)$ and agniastra (0.96, 1.08 and 1.10 plant $^{-1}$ ) conserved highest population of coccinellids, spiders and Microplitis coccons, respectively and were at par with untreated control (1.5, 1.3 and 1.34/ plant, respectively).

There was no significant effect of any of the cow based organics (Brahmastra, Agniastra and Neemastra) at 20\% dose after 5, 10 and 15 days of application. It clearly indicated that there was no adverse effect on the population of natural enemies in any of the cow based organic treatments including NSKE 5\%.

\section{Seed yield and Incremental Cost Benefit Ratio}

Highest pod yield of $885 \mathrm{~kg} \mathrm{ha}^{-1}$ was recorded in spray schedule comprising of acephate at first spray followed by second spray of thiodicarb and it was followed by NSKE @ $5 \%\left(742 \mathrm{~kg} \mathrm{ha}^{-1}\right)$, both are significantly different from each other but superior over all the other treatments (Table 2). Among the cow based organics, two scheduled sprays of agniastra @ 20\% recorded 474 kg/ha significantly followed by brahmastra @ 20\% (419 kg/ha). Two scheduled sprays of neemastra@20\% (392 kg ha ${ }^{-1}$ ) was at par with earlier treatment and also with untreated check which recorded lowest seed yield of $358 \mathrm{~kg} \mathrm{ha}^{-1}$.

Considering the Incremental Cost Benefit Ratio (ICBR), acephate at first spray followed by second spray of thiodicarb registered the incremental benefit of $21080 ₹$ ha $^{-1}$ with ICB ratio of 10.02 only. Although two scheduled sprays of NSKE@5\% recorded incremental benefit of $15380 ₹ \mathrm{ha}^{-1}$, it could record higher ICBR of 16.55 due to involvement of less treatment cost suggesting its suitability as one of the IPM components in reducing pest damage in castor. Among the cow based organics, two scheduled sprays of agniastra@ $20 \%$ recorded higher ICB ratio of 3.4 with incremental benefit of 4640 ₹ $\mathrm{ha}^{-1}$ compared to other organics. While two sprays of brahmastra@ 20\% recorded lower ICB ratio of 1.92 and neemastra@20\% recorded lowest ICB ratio of 0.60 with a meagre incremental benefit of $1360 ₹ \mathrm{ha}^{-1}$ over untreated check (Table 2).

The unilateral approach of controlling the castor pests by synthetic insecticides has dictated necessity for developing cost effective, eco-friendly and safe pest control strategies without using any chemical toxicants in the organic farming. Use of 
botanical pesticides for protecting crops from insect pests has assumed greater importance all over the world. Few workers have tested cow dung manure and liquid manure universally against insect pests other than castor crop and documented their potential against various pests. Shukla et al., (2003) revealed that fortification of cow urine with leaf extracts of various botanicals like neem, ipomea, annona and jatropa resulted in increased insecticidal property of the former against sucking pests of castor and capsule borer resulting in higher castor bean yield over control and proved an eco friendly alternative to hazardous pesticides. Similarly, reduction in insect population on different crops by using cow urine with various botanicals has also been reported by Patel et al., (2003) and Barapatre and Lingappa (2003).

The fermented products viz., neemastra, brahmastra and agniastra are reported to be possessing insecticidal properties against many pests including sucking pests (Subash Palekar, 2006). Patel et al., (2017) reported that brahmastra @20\% was found highly effective in suppressing the sucking pests of cotton viz., aphid, leafhopper, thrips and whitefly with highest seed cotton yield (27.74 $\left.\mathrm{q} \mathrm{ha}^{-1}\right)$ followed by agniastra (25.12 $\left.\mathrm{q} \mathrm{ha}^{-1}\right)$ and neemastra@20\% (23.99 q ha ${ }^{-1}$ ) without adverse effects on the natural enemies. According to Karthika (2013) spraying of brahmastra@5\% was found effective up to seventh day after application with good residual effect against the whitefly, mite, aphid, leafhopper and fruit borer in okra. Kavitha and Patil (2009) studied field performance of fermented plant products and aqueous extracts vividly showed that NSKE $5 \%$ spray recorded significantly less number of defoliator larvae and significantly highest yield $\left(20.80 \mathrm{q} \mathrm{ha}^{-1}\right)$ followed by agniastra $\left(19.67 \mathrm{q} \mathrm{ha}^{-1}\right)$ and brahmastra spray $(19.10 \mathrm{q}$ $\mathrm{ha}^{-1}$ ) in groundnut ecosystem which confirms the present results in castor. Field performance of cow based fermented organic products and aqueous extract of neem revealed that NSKE 5\% spray recorded significantly less number of defoliator larvae, leafhopper population and significantly highest yield (742 $\mathrm{kg} \mathrm{ha}^{-1}$ ) followed by agniastra (474 kg ha ${ }^{-1}$ ) and brahmastra spray (419 $\mathrm{kg} \mathrm{ha}^{-1}$ ) in castor ecosystem. All the organics were relatively safer against the natural enemies. Use of NSKE 5\% and agniastra in castor would become biological alternatives to insecticides for the control of major defoliator and sucking pests.

\section{References}

Barapatre A. and Lingappa S. 2003. Larvicidal and antifeedant activity of indigenous plant protection practices for Helicoverpa armigera (Hub.). In: Proceedings of National Symposium on Frontier Areas of Entomological Research, November 5-7, 2003, pp. 335-336.

Gomez K A and Gomez A A. 1984. Statistical Procedures for Agricultural Research, $2^{\text {nd }}$ Edition, A Wiley Interscience Publication, John Wiley and Sons, New York, pp. 302-307.

Karthika C 2013. Development of technologies for organic bhendi (Abelmoschus esculentus L.Moench) seed production. Ph.D. Thesis submitted to Tamil Nadu Agricultural University, Coimbatore.

Kavitha A S and Patil R K 2009. Ecofriendly practices against major pests in different cropping systems with special reference to groundnut. Abstracts of Thesis, Karnataka Journal of Agricultural Sciences, 22 (5): 1154- 1205.

Kavitha A S 2009. Eco- friendly practices against major pests in different cropping systems with special 
reference to groundnut. M.Sc (Ag) thesis submitted to the University of Agricultural Sciences, Dharwad.

Mukesh B, Naveen Kumar N and Acharya M F 2016. Bioefficacy of modern insecticides against Spodoptera Litura Fabricius on Castor. International Journal of Agriculture Innovations and Research, 4 (4): 789-795

Patel P S, Shukla N P and Patel G M 2003. Enhancing insecticide properties of cow urine against sucking pests of cotton. In: Proceedings of National Symposium on Frontier Areas of Entomological Research, 5-7 November, 2003 pp-227.

Patel R D, Bharpoda T M, Borad P K, Bhatt N A and Mahida R D 2017. Efficacy of different bio-pesticides against sucking pets of $B t$ Cotton. AGRES -
An International e-Journal, 6 (1): 171180

Shilpakala V and Muralikrishna T 2016. Evaluation of New Insecticide Molecules Against Lepidopteran Pests in Castor (Ricinus communis L.). International Journal of Agricultural Science and Research, 6 (6): 29-36.

Shukla N P, Patel P S and Pate G M 2003. Fortification of cow urine for enhancing insecticidal properties against pests of castor. In : Proceedings of National Symposium on Frontier Areas of Entomological Research, 5-7 November, 2003, p. 216.

Subash Palekar, 2006, Naisargika Krishi, pp. 61-63.

\section{How to cite this article:}

Suneel Kumar, G. V. and Sarada, O. 2020. Evaluation of Cow Based Fermented Organic Products for Non-insecticidal Pest Management in Castor. Int.J.Curr.Microbiol.App.Sci. 9(10): 292-300. doi: https://doi.org/10.20546/ijcmas.2020.910.037 